\title{
Binary post-AGB stars as tracers of stellar evolution
}

\author{
Hans Van Winckel
}

\begin{abstract}
In this chapter the focus is on the properties of post-Asymptotic Giant Branch (post-AGB) stars in binary systems. Their Spectral Energy Distributions (SEDs) are very characteristic: they show a near-infrared excess, indicative of the presence of warm dust, while the central stars are too hot to be in a dust-production evolutionary phase. This allows for an efficient detection of binary post-AGB candidates. It is now well established that the near-infrared excess is produced by the inner rim of a stable dusty disc that surrounds the binary system. These discs are scaled-up versions of protoplanetary discs and form a second generation of stable Keplerian discs. They are likely formed during a binary interaction process when the primary was on ascending the AGB. I will summarise what we have learned from the observational properties of these post-AGB binaries. The impact of the creation, lifetime and evolution of the circumbinary discs on the evolution of the system are yet to be fully understood.
\end{abstract}

\subsection{Introduction}

The final evolution of low- and intermediate-mass stars $\left(0.8-8 \mathrm{M}_{\odot}\right)$ is a rapid transition from the Asymptotic Giant Branch (AGB) over the post-AGB phase towards the Planetary Nebulae (PNe) stage. When most of the stellar envelope is removed by an intense massloss rate (up to $10^{-4} \mathrm{M}_{\odot} / \mathrm{yr}$ ) at the end of the AGB, the star enters the post-AGB phase of stellar evolution. During this phase the remaining stellar envelope begins to shrink and the effective temperature starts to increase with almost constant luminosity. If the temperature increases on a timescale shorter than the dispersion time of the matter previously ejected by the star, a PNe will appear, as the result of the ionization of the circumstellar shell (Balick and Frank 2002, Herwig, 2005). During the AGB to PNe transition, some objects show a resolved reflection nebula and these are referred to as proto-planetary nebula or pre-planetary nebula (Sahai et al. 2007). These proto-planetary nebulae are only a subset of post-AGB stars, as the latter also includes objects that will not ionise their ejected matter as it is too dispersed. It is fair to say that the samples of proto-planetary nebulae and post-

This material has been published in The Impact of Binaries on Stellar Evolution, Beccari G. \& Boffin H.M.J. (Eds.). This version is free to view and download for personal use only. Not for re-distribution, re-sale or use in derivative works. (C) 2018 Cambridge University Press. 
AGB stars in general, do not connect very well on evolutionary tracks with the AGB stars, nor with the PNe. The diversity seen in individual examples is not well understood in the framework stellar evolution theory (e.g.Van Winckel, 2003).

One of the most important research questions regarding the final evolution of low-and intermediate-mass stars, is the impact of binarity (see recent review by De Marco and Izzard, 2017, and references therein). Also this book provides ample illustration of research results focussing on this question. Binary interaction alters the intrinsic properties of the evolved star (such as: pulsations, mass-loss, dust-formation, circumstellar envelope morphology etc.) and plays a dominant role in determining its ultimate fate. A plethora of peculiar objects ranging from the spectacular thermonuclear novae, supernovae type Ia, sub-luminous supernovae, gravitational wave sources, etc. to less energetic systems such as sub-dwarf B stars, barium stars, cataclysmic variables, bipolar PNe, etc., result from mass transfer in binary stars.

During evolution of the low- and intermediate-mass star, binary interactions will increase when one of the binary components evolves to giant dimensions. The dynamical behaviour of this interaction is determined by the balance between the Roche Lobe and the stellar radius of the red giant. Binaries with a main sequence separation of less than about two astronomical units, will be tidally captured somewhere on the Red Giant Branch (RGB) or AGB. When unstable mass transfer ensues, a common envelope (CE) event occurs, resulting in a dramatic shortening of the orbital separation. This might lead to a complete stellar merger but, if this is avoided, the final period of these binaries after the giant phase is typically in the order of days, depending on the envelope's binding energy. When the initial orbit is wider, the interaction is thought to be via wind accretion or wind shaping and the primary component will evolve off the giant branch with a very wide orbit. When the originally lower mass component evolves also onto the giant branch, interaction may take place again, but this time with a compact companion. This theoretical scenario is borne out by population-synthesis models (e.g. Han et al. 2002, 2003). The populationsynthesis model normalised to the ellipsoidal binaries on the red giant phase, predicts the final period distribution of evolved binaries to be bi-modal in which the $\mathrm{CE}$ channel results in short-period binaries ( $\mathrm{P} \sim 1$ day) and the wind accretion channel results in wider systems ( $\mathrm{P}>1000$ days) (Nie et al. 2012). The orbital periods of around 1000 days are least predicted and lie in the middle of this bi-modal distribution (see also Chapter by Pols in this book).

This is, however, in stark contrast to what is observed in post-AGB binaries and here we focus on the observed properties of these optically bright objects. The reasons for this strong discrepancy between the observed and predicted period distributions are twofold. Firstly, a wide range of binary interactions are not understood from first principles or are even missing in the binary evolution models. The theoretical models are therefore subject to many uncertainties like the efficiency of envelope ejection (e.g. Toonen and Nelemans, 2013); the unknown physical description of the common-envelope phase (e.g. Izzard et al. 2012; Ivanova et al. 2013); the postulated increase of the mass-loss prior to contact as to lower the envelope binding energy (e.g. Chen et al., 2011; Abate et al. 2013); the badly 
understood impact of radiation pressure on the shape of the classical Roche potential (Dermine et al. 2009); the assumed mass transfer efficiency and its orbital phase dependency. Secondly, there is also an observational challenge as many parameters involved in predicting the outcome of the binary evolution channels are not well constrained due to the lack of observational data, where detailed studies of individual objects prevail over systematic and time-consuming more complete surveys.

In this chapter we focus on binary post-AGB stars and their observational properties. In Section 1.2 we focus on their Spectral Energy Distribution (SED) as this turned out to be very characteristic. We report on the orbital properties in Section 1.3 and review all observational evidence for the presence of stable circumbinary discs as a common feature of all the binaries in Section 1.4 Jets and their origin form the core of Section 1.5. The impact of the feedback from the disc on the primary is given in Section 1.6 We end in Section 1.7 by naming a few challenges ahead to come to a better understanding of binary stellar evolution of low-and intermediate mass stars.

\subsection{SED}

While the first binary post-AGB stars were serendipitously detected (e.g. Waelkens et al. 1996) it turned out that their SEDs had some common but distinct properties. This is illustrated in Figure 1.1 where a typical SED of a post-AGB binary is displayed. The energetics display a clear near-infrared excess by thermal emission of dust, indicating that this circumstellar dust must be close to the central star, near sublimation temperature. The peak of the dust excess is around $10 \mu \mathrm{m}$ and at the long wavelength tail, the spectral index follows the Rayleigh jeans slope up to submm wavelengths. It is now well established that these features in the SED indicate the presence of a stable compact disc in the system (see Section 1.4). We call these type of SEDs, disc-type SEDs.

These specific SED characteristics, in combination with the availability of all-sky infrared surveys of IRAS, WISE and AKARI allowed for efficient searches for similar Galactic systems (e.g. De Ruyter et al., 2006; Gezer et al., 2015) and in total about 85 Galactic such sources have been identified by now. These SEDs are in clear contrast to the ones of optically bright post-AGB stars with detached shells: the shell of gas and dust, which was expelled when the object was still on the AGB, is cooling and expanding resulting in an SED without a near-IR excess and a peak in the SED sypically around 30-60 $\mu \mathrm{m}$ (Van Winckel, 2003).

Moreover, the SPITZER surveys in the Large and Small Magellanic Clouds (Meixner et al. 2006; Bolatto et al. 2007, Gordon et al. 2011) allowed for a systematic approach in the search for post-AGB stars also in these galaxies. After a photometric selection (van Aarle et al., 2011, Kamath et al. 2014), a spectral survey was performed to locate similar evolved sources and to differentiate them from interlopers (Kamath et al., 2014, 2015). The objects with distinct near-IR excess represent more than half of the population of optically bright post-AGB stars. Objects with similar SEDs also appear at lower luminosities, in- 


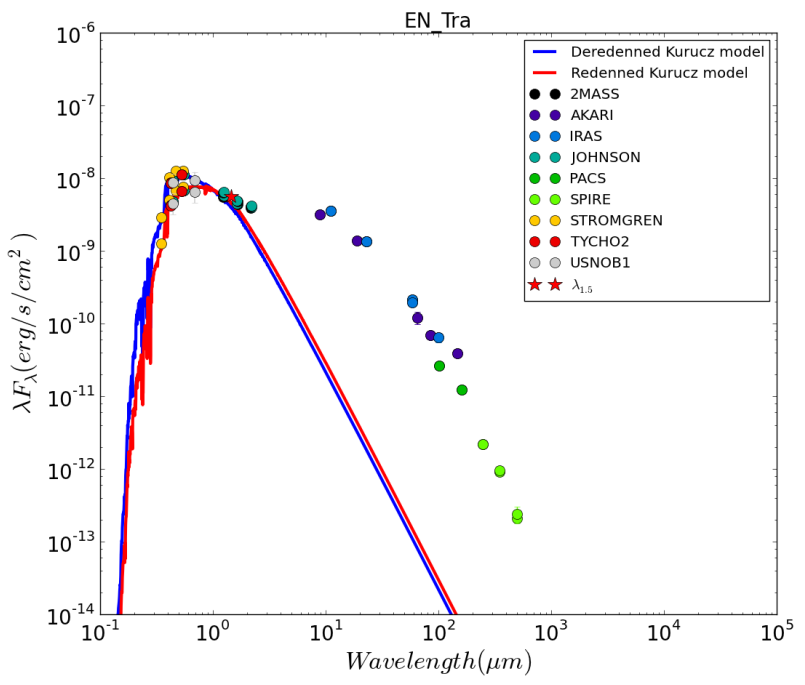

Figure 1.1 The SED of 89Her (Hillen et al. 2013) as an example of an optically bright post-AGB binary.

dicating that the central evolved star is a post-Red Giant Branch (post-RGB) stars, rather than post-AGB stars (Kamath et al. 2016).

We conclude that within the known samples of the rare post-AGB stars, disc SEDs are very frequently detected and this both in the Galaxy and the Magellanic clouds.

\subsection{Orbital Properties}

The binary nature of individual objects (Van Winckel et al., 1995; Waelkens et al., 1996, Gonzalez and Wallerstein, 1996; Maas et al., 2002) inspired a systematic monitoring programme of Galactic evolved sources using our own HERMES spectrograph (Raskin et al. 2011) mounted on the Flemish 1.2m Mercator telescope. This has resulted in the discovery of many evolved binaries with unexpected periods between 100 and 2000 days within the sample of sources with disc SEDs (e.g. Van Winckel et al. 2009, Gorlova et al., 2012, Gezer et al., 2015; Manick et al., 2017). The $a \sin i$ is typically $\sim 1$ AU. The luminous evolved component has a likely unevolved companion with a very minor contribution to the energy budget (e.g. Van Winckel et al., 2009). The Figure 1.2 is illustrative of this. One of the most striking results is a clear lack of spiralled-in systems at short periods in the order of days. This is not a observational bias: these would be the most easy to detect in radial velocity monitoring programmes. Another striking observation is the rare occurrence of zero-eccentricity systems as many objects have significant eccentricities. 


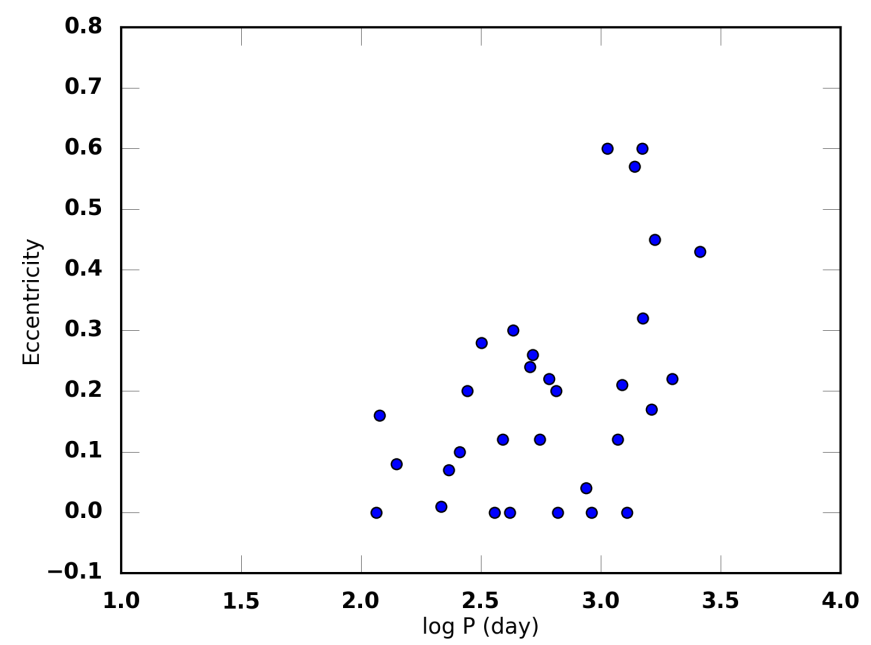

Figure 1.2 The e- $\log (\mathrm{P})$ diagram of post-AGB binaries.

The global picture that emerges from the orbital elements is that the star evolved in a system which is too small to accommodate a full grown RGB or AGB star. During a poorly understood phase of strong interaction, the system did not suffer a dramatic spiral in. The observed orbits (see Figure 1.2) show that the common envelope was either very rapidly expelled or somehow avoided. We postulate that this binary interaction phase truncated the AGB or RGB evolution. All the $\sim 30$ orbits found till now and their non-zero eccentricities fall in a period regime not predicted by standard binary channels. Moreover, the tidal circularisation was not effective and most objects have distinct eccentric orbits. Eccentricity pumping mechanisms need to be invoked to explain the eccentricities. As the dust sublimation radii for these sources are well beyond the orbit, the dusty discs are circumbinary.

Finding binaries among post-AGB stars with an expanding shell (shell-sources), on the contrary, turned out to be a cumboursome challenge: despite radial-velocity monitoring efforts for decades, confirmed spectroscopic binaries were not detected yet in post-AGB stars with such detached shells which puts strong constraints on the companion mass and/or possible orbits (Hrivnak et al., 2011, 2017). Interestingly, these detached shells are often resolved and show bipolar and even multipolar geometries (Hrivnak et al., 2017), which are often associated with binary interaction physics. If at all, these shell objects are likely very wide systems.

Spectroscopic binaries in post-AGB stars are uniquely connected to their SED properties: almost all binaries known till now have disc-SEDs. Very few exceptions exist and these have a very small dust excess which seems to be the relic of the circumbinary disc. 


\subsection{Discs: Resolved from the inner Edge to the outer Radius}

\subsubsection{Near and Mid-IR Interferometry}

It is now well established that this type of disc-SED, is a good observational proxy for the presence of a stable compact circumbinary disc. As the infrared emission is compact and the objects are typically at a distance of several kpc, interferometric techniques are needed to resolve them. Applications of high-spatial-resolution techniques with limited uv-coverage (Deroo et al., 2006, 2007; Hillen et al., 2013, 2014, 2015, 2017) are unveiling the very compact infrared emission regions. There is by now ample evidence that these circumbinary discs show many similarities with the passive protoplanetary discs (PPDs) around young stars (e.g. Dullemond and Monnier, 2010, Menu et al., 2015).

The spatial information obtained by interferometric experiments are combined with detailed physical modelling via radiative transfer calculations, in which the transport of radiation in three dimensions is treated self-consistently. The vertical scale of the disc is computed under the assumption of hydrostatic equilibrium. For the opacity calculations, the opacities of silicates are typically used (Min et al. 2007) with a variable size distribution of the grains. Turbulent mixing and vertical settling of grains can be included self-consistently (Mulders and Dominik. 2012), as it strongly affects the structure of the disc, and therefore the observables. The good agreement between the best models and the interferometric observations in these references above gives strong support to the physical interpretation of the circumstellar material: the structure is that of a dusty settled disc. The best-fit grain size distribution shows that grain growth is significant. Sub-micron-sized particles hardly contribute to the total opacity in this disc, while mm-sized grains need to be included to fit the sub-mm fluxes.

A first interferometric imaging experiment, which is possible provided the UV-plane is covered extensively, was presented in Hillen et al. (2016). Thanks to the unprecedented spatial resolution, all important building blocks (circumbinary disc, central binary, accretion disc around the companion) could be spatially resolved and their flux contribution could be quantified which is illustrated in Figure 1.3 . The most apparent is the inner rim of the circumbinary disc, which is indeed at the sublimation radius of the star. For clarity the central bright star, which is unresolved, is removed from the image. The companion itself is too faint to contribute significantly to the total H-band flux, but it is the accretion disc around the companion which is resolved as an additional contributor to the center of the image, slightly displaced from the luminous post-AGB star. The physical model of the circumbinary disc assumes a settled disc in hydrostatic equilibrium, with an axisymmetric surface density. While the model fits many (interferometric) observables, the real data shows more structure.

Just like for pre-main-sequence stars across a wide range of mass and luminosity (Dullemond and Monnier, 2010; Kraus et al., 2010), spectro-interferometry proves very powerful to study the physics of settled discs. 

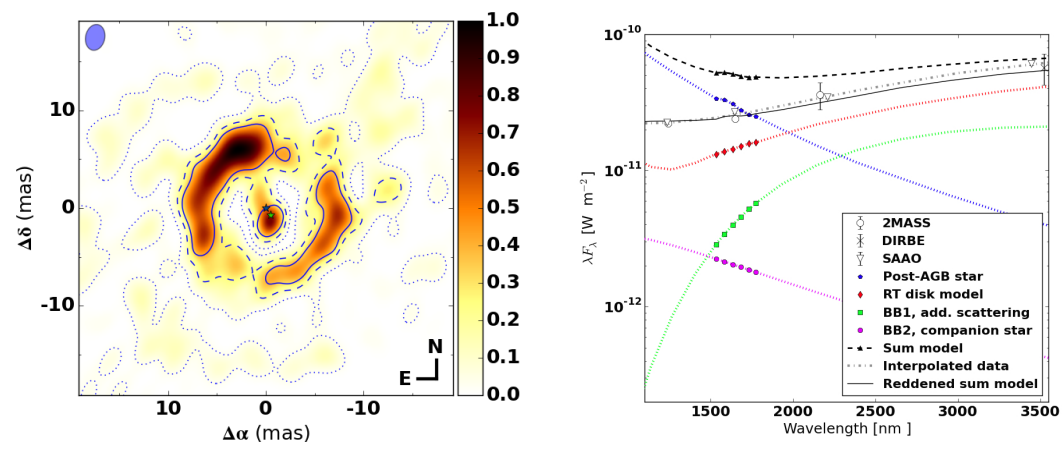

Figure 1.3 The interferometric image in the H-band of IRAS08544-4431 on the left. The central luminous post-AGB star is removed for clarity (blue star). The inner rim of the circumbinary disc is well resolved and the off-centre flux contribution is interpreted as coming from the accretion disc around the companion (Hillen et al. 2016). On the right, the dissection of the SED is shown with all spatially resolved contributing components.

\subsubsection{CO Interferometry}

The most conclusive evidence for stability comes from the detection of resolved Keplerian velocity fields in three systems so far: the Red Rectangle, AC Her and IW Car. These were spatially resolved in CO using the ALMA and the Plateau De Bure interferometers (Bujarrabal et al., 2013, 2015, 2017). Moreover, for 89 Her the inner CO disc is found to be not resolved but likely in Keplerian rotation as well (Bujarrabal et al., 2007).

In addition to the Keplerian velocity, a slowly expanding component was resolved in the maps of the Red Rectangle, 89 Her and IW Car, showing a bipolar low-velocity outflow. Given its structure and kinematics, these outflows likely originate from the disc. The total angular momentum of the disc of the Red Rectangle is significant and suggests that, if the orbit is the source of angular momentum, the binary should have shrunk considerably (Bujarrabal et al. 2016). By comparing the total mass of the CO disc with the mass-loss as detected in $\mathrm{CO}$, the estimated lifetimes are between $\sim 8000$ and $10000 \mathrm{yr}$ (Bujarrabal et al. 2016, 2017).

Single-dish observations confirm that rotation must be widespread among other disc sources as well: the $\mathrm{CO}$ rotational lines are very narrow indicating that Keplerian rotation rather than expansion is detected in all the sources of the survey (Bujarrabal et al., 2013). Clearly more systems need to be investigated in detail.

\subsubsection{Longevity}

The discs have other distinct observational features which are interpreted as indicators of longevity. Infrared spectroscopic observations of dust features show that strong dust- 
grain processing is needed to explain the profiles. The processing leads to a high degree of crystallinity in the silicate bands (e.g. Molster et al., 2002; Gielen et al., 2007, 2011).

It is remarkable that in all systems, Galactic and from the Maggelanic clouds alike, the dust is oxygen rich and Mg-rich endmembers of the silicates prevail. Aditionally, some objects show a carbon-rich component like the Extended Red Emission (ERE) in the Red Rectangle (e.g. Cohen et al., 2004) and PAH emission in a minority of objects.

Another indicator for longevity, is the presence of large grains. In order too explain the contrast and width of the silicate bands (e.g. Gielen et al. 2007. 2011: Arneson et al. 2017), micron sized grains are needed. The presence of even larger grains, to the $\mathrm{cm}$ regime, are needed to explain the spectral index at sub-mm wavelengths (e.g. Jura et al., 1997, De Ruyter et al., 2005, Gielen et al., 2007; Hillen et al., 2014, 2015).

\subsection{Outflows and Jets}

Another important structural component is detected only indirectly. This is best illustrated by showing orbital phase-resolved high-resolution spectra (Figure 1.4). the $\mathrm{H}_{\alpha}$ profile turns into a P-Cygni profile only when the unseen companion is in front of the luminous primary which is the post-AGB star. The interpretation of this behaviour is that a high velocity outflow or jet, originates around the companion and is only detected when continuum photons of the primary are scattered out of the line-of-sight (Thomas et al., 2013; Gorlova et al. 2012, 2015). When a good phase coverage is reached, the wide opening angle as well as the latitudinally dependent velocity law can be deduced from the spectra (Thomas et al. 2013; Bollen et al., 2017). For the objects which are well studied, the outflow velocity is larger in the polar direction, with a high-velocity and low density on the jet axes, and a lower velocity, denser outflow at the conical edge. The deprojected velocity corresponds to the escape velocity of a main sequence star, rather than from a compact object.

The jets are not strongly collimated, but are launched likely from a circum-companion accretion disc. This disc is seen outside conjunction via the shell-profiles of the $\mathrm{H}_{\alpha}$ emission, which can be used to map in velocity space, via Doppler tomography (Bollen et al. 2017). Whether the circum-companion accretion disc is fed by direct mass transfer from the primary, or via accretion from the circumbinary disc is not constrained yet.

While modelled and published only in a limited number of systems till now, similar orbital phase-dependent Halpha profiles are now commonly observed in post-AGB binaries (see Figure 1.4). When a system is seen nearly pole-on, the P-Cygni line-profile is seen at all orbital phases as the wide jet is always in the line-of-sight. Post-AGB binaries provide ideal test beds to study jet formation and launching mechanisms over a wide range of orbital periods and mass accretion regimes. 

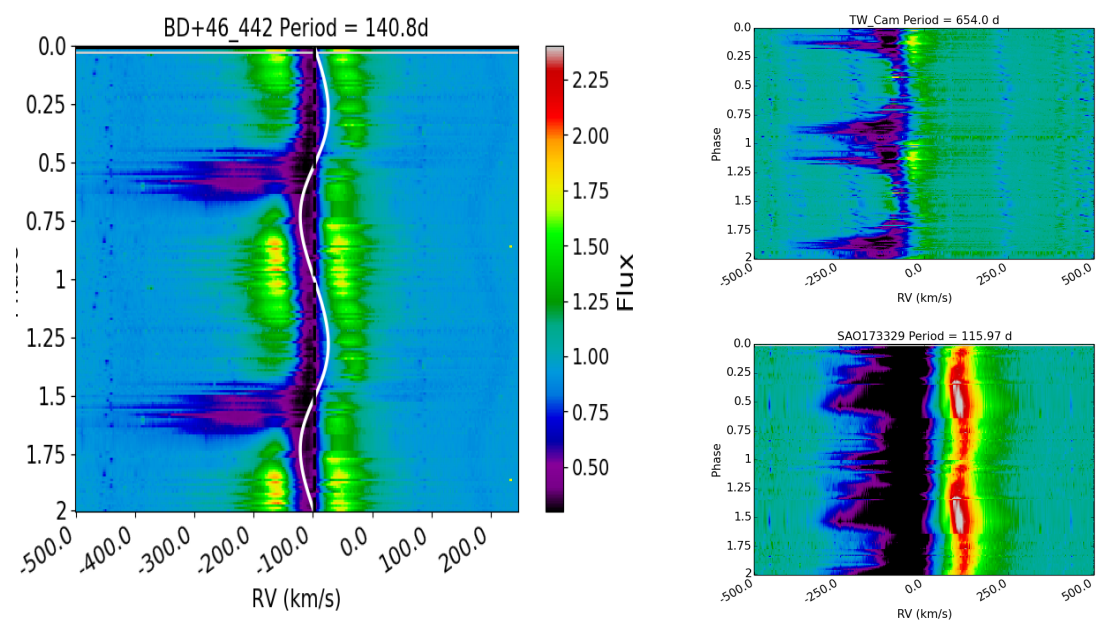

Figure 1.4 Dynamic H-alpha profiles folded on the orbital period. On the left, the data of BD $+46^{\circ} 442$ (Bollen et al. 2017) folded on its 140.7 days orbital period. The full line represents the movement of the primary. The absorbtion at high velocity appears when the companion is in front of the primary (phase 0.5). On the right two other illustrative examples. At the top, the $\mathrm{H}_{\alpha}$ profiles of the RV Tauri pulsator TW Cam are displayed, folded on the orbital period of 654 days. The pulsations are seen in the photospheric lines (Manick et al. 2017). The absorption component appears when the companion is in front (phase 1.0). The bottom shows the profiles of SAO173329 with an orbital period of 116 days. Here we look almost pole-on which makes that the high velocity outflow is always in the line-of-sight to the primary.

\subsection{Feedback from Circumbinary Discs}

Many post-AGB stars display a chemical anomaly in their photosphere called 'depletion' (Van Winckel, 2003; Rao and Giridhar, 2014; Venn et al., 2014). The abundance trends in such atmospheres resemble the gas phase of the interstellar medium: refractory elements are underabundant, while volatiles retain their original abundances (see Figure 1.5. Good tracers of depletion are the $[\mathrm{S} / \mathrm{Ti}]$ and $[\mathrm{Zn} / \mathrm{Ti}]$ or $[\mathrm{Zn} / \mathrm{Fe}]$ abundance ratios. The $s$-process elements are also refractory (Lodders, 2003) so the eventual enrichment of AGB nucleosynthesis products is masked by the depletion process.

The process to acquire this chemical anomaly is not completely understood yet. Dust formation leads to a chemical fractionation in the circumstellar environment. To obtain a depleted photosphere, the radiation pressure on circumstellar dust grains must fractionate the dust from the gas. The cleaned gas is then re-accreted on to the stellar surface.

Such depletion patterns are intimately linked to the presence of a disc around the postAGB star, and indicate that gas, cleaned from the refractory elements by dust formation, is accreted to the primary (e.g. Giridhar et al., 2005, Gezer et al. 2015, and references therein). The depletion process can be very efficient and in some case the original Fe content is reduced to $[\mathrm{Fe} / \mathrm{H}]=-3$ to -4 ., while the non-refractory elements like $\mathrm{S}$ and $\mathrm{Zn}$ 


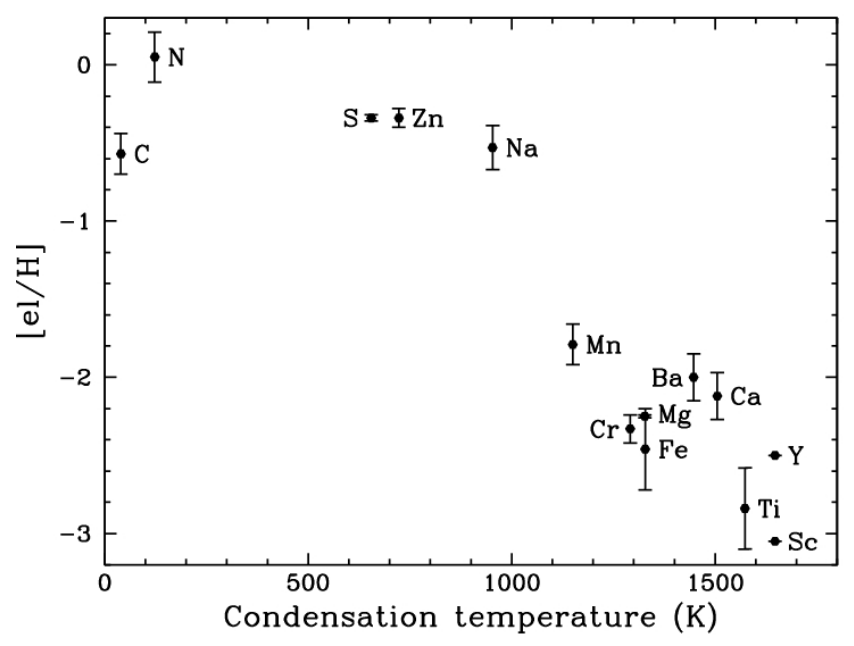

Figure 1.5 A depleted photosphere has a chemical anomaly: the photopheric elemental abundances correlate with the dust condenstationtemperatures. (Figure give the abundances of MACHO82.8405.15 adapted from (Gielen et al. 2009)).

retained their original abundances (Van Winckel et al. 1995, Venn et al. 2014). Depleted atmospheres are also found in post-AGB stars in the LMC and SMC (Reyniers and Van Winckel, 2007, Gielen et al., 2009).

The longevity of the disc (Waters et al. 1992), seem to be a needed but not sufficient condition for the process to occur. As shown in a more systematic comparison in Gezer et al. (2015), depleted photospheres are striclty limited to objects with a disc. For some exceptions, there is good observational evidence of a former disc. If the accretion of mass is similar or larger than the envelope reduction due to external mass-loss and core-growth, the post-AGB lifetime will be lengthened.

\subsection{Conclusions}

The specific SED charateristics of established binary post-AGB stars, allow for a very efficient search for similar disc-SEDs among the evolved optically bright stars. Moreover, detailed and long-term radial velocity monitoring programmes have shown that the central stars with disc-SEDs are indeed binaries with typical orbital periods between 100 and 2000 days (Figure 1.2), which are orbits least predicted by stellar binary evolution models. No spiralled-in systems are found, despite the orbital sizes which are too small to accomodate an AGB star. While spiralled-in systems are commonly observed in central stars of PNe (e.g. Jones and Boffin 2017), post-AGB stars with discs evolve on tracks which avoid 
spiralling-in. Post-AGB binaries are by no means the only evolved binaries with orbits that are not well understood (see e.g. chapter by Pols).

The discs around evolved binaries are scaled-up versions of protoplanetary discs, because the ratio of luminosity versus mass is much higher. They have a physically larger dust-free inner region of several astronomical units. It is remarkable that a hydrostatic model, dominated by dust sublimation physics can represent the morphology and energetics of the circumbinary disc even better than it does for discs around young stars, for which these models were originally developed (Hillen et al., 2017). Distances are typically poorly constrained, except for the disc sources in the LMC and SMC but for the latter, orbital properties are as yet not derived (Kamath et al., 2014, 2015). This does hamper the comparison with binary models. Constraining the distances will become possible when GAIA accumulates enough data that enables differentiation between parallax movements and orbital movements.

There is increasing evidence that the presence of a circumbinary disc has a lasting effect on the dynamical evolution of the binary orbit, as resonant coupling between the binary and the disc can pump-up the eccentricity (Artymowicz et al. 1991, Lubow, 2010, Dermine et al. 2013; Vos et al. 2015). This provides a way to explain the commonly observed large eccentricities. The integrated effect depends strongly on the mass and longevity of the disc, as well as on the radial mass distribution within the disc. Quantification of this eccentricity-pumping effect is hence only possible when the evolution and timescale of the discs are understood. Moreover the total angular momentum stored in the discs are, so far, determined in a few systems only (Bujarrabal et al., 2017).

Jets are seen as a major shaping agent in PNe (Balick and Frank, 2002, De Marco and Izzard, 2017) and are thought to shape the multipolar and often complex reflection nebulae in proto-planetary stars (Sahai et al., 2007). Grazing jets (Soker, 2017; Shiber et al., 2017) are potentially important for avoiding common-envelope phase in many binary systems. Despite their importance, jet physics and launching mechanisms are still poorly understood. The optically bright post-AGB binaries offer ideal test beds for jets and their creation processes. The circumcompanion accretion discs where they are launched can be fed either by the primary, or by the circumbinary dusty disc or both. The reflection nebulae, which are shell-sources, are often very multi-polar and structured, but for these, the monitoring data complies only with either single stars, very wide systems, or very low-mass companions (Hrivnak et al. 2017). These shell systems are very different than the objects with disc-SEDs.

We conclude that stable circumbinary discs are integral component of many post-AGB binaries and are likely playing a lead role in the evolution of the systems. We can postulate the disc creation, evolution and evaporation play a lead role in all systems with a former AGB star. Detailed studies of the structure and evolution of these discs and their impact on the orbital evolution are therefore badly needed. 


\subsection{Acknowledgements}

It is a pleasure to acknowledge the many colleagues, post-docs and $\mathrm{PhD}$ students which contributed recently to the research on post-AGB binaries: Christoffel Waelkens, Devika Kamath, Peter Wood, Valentin Bujarrabal, Orsola De Marco, Michel Hillen, Alain Jorissen, Sophie van Eck, Lionel Siess, Onno Pols, Gijs Nelemans, Bruce Hrivnak, Brent Miszalski, Rajeev Manick, Ana Escorza, Shreeya Shetye, Glenn-Micheal Oomen, Dylan Bollen, Ilknur Gezer and Joonas Sario.

\section{References}

Abate, C., Pols, O. R., Izzard, R. G., Mohamed, S. S., and de Mink, S. E. 2013. Wind Roche-lobe overflow: Application to carbon-enhanced metal-poor stars. $A \& A$, 552(Apr.), A26.

Arneson, R. A., Gehrz, R. D., Woodward, C. E., Helton, L. A., Shenoy, D., Evans, A., Keller, L. D., Hinkle, K. H., Jura, M., Lebzelter, T., Lisse, C. M., Rushton, M. T., and Mizrachi, J. 2017. A SOFIA FORCAST Grism Study of the Mineralogy of Dust in the Winds of Proto-planetary Nebulae: RV Tauri Stars and SRd Variables. ApJ, 843(July), 51.

Artymowicz, P., Clarke, C. J., Lubow, S. H., and Pringle, J. E. 1991. The effect of an external disk on the orbital elements of a central binary. ApJ, 370(Mar.), L35-LL38.

Balick, B., and Frank, A. 2002. Shapes and Shaping of Planetary Nebulae. ARA\&A, 40, 439-486.

Bolatto, A. D., Simon, J. D., Stanimirović, S., van Loon, J. T., Shah, R. Y., Venn, K., Leroy, A. K., Sandstrom, K., Jackson, J. M., Israel, F. P., Li, A., Staveley-Smith, L., Bot, C., Boulanger, F., and Rubio, M. 2007. The Spitzer Survey of the Small Magellanic Cloud: $\mathrm{S}^{3} \mathrm{MC}$ Imaging and Photometry in the Mid- and Far-Infrared Wave Bands. ApJ, 655(Jan.), 212-232.

Bollen, D., Van Winckel, H., and Kamath, D. 2017. Jet creation in post-AGB binaries: the circum-companion accretion disc around $\mathrm{BD}+46^{\circ} 442$. ArXiv e-prints, Aug.

Bujarrabal, V., Van Winckel, H., Neri, R., Alcolea, J., Castro-Carrizo, A., and Deroo, P. 2007. The nebula around the post-AGB star 89 Herculis. A\&A, 468(June), L45-L48.

Bujarrabal, V., Castro-Carrizo, A., Alcolea, J., Van Winckel, H., Sánchez Contreras, C., Santander-García, M., Neri, R., and Lucas, R. 2013. ALMA observations of the Red Rectangle, a preliminary analysis. $A \& A, \mathbf{5 5 7}$ (Sept.), L11.

Bujarrabal, V., Castro-Carrizo, A., Alcolea, J., and Van Winckel, H. 2015. Detection of Keplerian dynamics in a disk around the post-AGB star AC Herculis. A\&A, 575(Mar.), L7.

Bujarrabal, V., Castro-Carrizo, A., Alcolea, J., Santander-García, M., van Winckel, H., and Sánchez Contreras, C. 2016. Further ALMA observations and detailed modeling of the Red Rectangle. A\&A, 593(Sept.), A92.

Bujarrabal, V., Castro-Carrizo, A., Alcolea, J., Van Winckel, H., Sánchez Contreras, C., and Santander-García, M. 2017. A second post-AGB nebula that contains gas in rotation and in expansion: ALMA maps of IW Carinae. $A \& A, \mathbf{5 9 7}$ (Jan.), L5.

Chen, X., Han, Z., and Tout, C. A. 2011. Tidally Enhanced Stellar Wind: a Way to Make the Symbiotic Channel to Type Ia Supernova Viable. ApJ, 735(July), L31. 
Cohen, M., Van Winckel, H., Bond, H. E., and Gull, T. R. 2004. Hubble Space Telescope Imaging of HD 44179, The Red Rectangle. AJ, 127(Apr.), 2362-2377.

De Marco, O., and Izzard, R. G. 2017. Dawes Review 6: The Impact of Companions on Stellar Evolution. Publ. Astron. Soc. Australia, 34(Jan.), 1-40.

De Ruyter, S., Van Winckel, H., Dominik, C., Waters, L. B. F. M., and Dejonghe, H. 2005. Strong dust processing in circumstellar discs around 6 RV Tauri stars. Are dusty RV Tauri stars all binaries? A\&A, 435(May), 161-166.

De Ruyter, S., Van Winckel, H., Maas, T., Lloyd Evans, T., Waters, L. B. F. M., and Dejonghe, H. 2006. Keplerian discs around post-AGB stars: a common phenomenon? $A \& A$, 448(Mar.), 641-653.

Dermine, T., Jorissen, A., Siess, L., and Frankowski, A. 2009. Radiation pressure and pulsation effects on the Roche lobe. A\&A, 507(Nov.), 891-899.

Dermine, T., Izzard, R. G., Jorissen, A., and Van Winckel, H. 2013. Eccentricity-pumping in post-AGB stars with circumbinary discs. A\&A, 551(Mar.), A50.

Deroo, P., van Winckel, H., Min, M., Waters, L. B. F. M., Verhoelst, T., Jaffe, W., Morel, S., Paresce, F., Richichi, A., Stee, P., and Wittkowski, M. 2006. Resolving the compact dusty discs around binary post-AGB stars using N-band interferometry. A\&A, 450(Apr.), 181-192.

Deroo, P., Acke, B., Verhoelst, T., Dominik, C., Tatulli, E., and Van Winckel, H. 2007. AMBER and MIDI interferometric observations of the post-AGB binary IRAS 085444431: the circumbinary disc resolved. $A \& A$, 474(Nov.), L45-L48.

Dullemond, C. P., and Monnier, J. D. 2010. The Inner Regions of Protoplanetary Disks. ARA\&A, 48(Sept.), 205-239.

Gezer, I., Van Winckel, H., Bozkurt, Z., De Smedt, K., Kamath, D., Hillen, M., and Manick, R. 2015. The WISE view of RV Tauri stars. MNRAS, 453(Oct.), 133-146.

Gielen, C., Van Winckel, H., Waters, L. B. F. M., Min, M., and Dominik, C. 2007. Dustgrain processing in circumbinary discs around evolved binaries. The RV Tauri spectral twins RU Centauri and AC Herculis. A\&A, 475(Nov.), 629-637.

Gielen, C., Van Winckel, H., Reyniers, M., Zijlstra, A., Lloyd Evans, T., Gordon, K. D., Kemper, F., Indebetouw, R., Marengo, M., Matsuura, M., Meixner, M., Sloan, G. C., Tielens, A. G. G. M., and Woods, P. M. 2009. Chemical depletion in the Large Magellanic Cloud: RV Tauri stars and the photospheric feedback from their dusty discs. $A \& A, \mathbf{5 0 8}$ (Dec.), 1391-1402.

Gielen, C., Bouwman, J., van Winckel, H., Lloyd Evans, T., Woods, P. M., Kemper, F., Marengo, M., Meixner, M., Sloan, G. C., and Tielens, A. G. G. M. 2011. Silicate features in Galactic and extragalactic post-AGB discs. A\&A, 533(Sept.), A99.

Giridhar, S., Lambert, D. L., Reddy, B. E., Gonzalez, G., and Yong, D. 2005. Abundance Analyses of Field RV Tauri Stars. VI. An Extended Sample. ApJ, 627(July), 432-445.

Gonzalez, G., and Wallerstein, G. 1996. ST Pup: a binary Type II Cepheid with a peculiar chemical composition. MNRAS, 280(May), 515-536.

Gordon, K. D., Meixner, M., Meade, M. R., Whitney, B., Engelbracht, C., Bot, C., Boyer, M. L., Lawton, B., Sewiło, M., Babler, B., Bernard, J.-P., Bracker, S., Block, M., Blum, R., Bolatto, A., Bonanos, A., Harris, J., Hora, J. L., Indebetouw, R., Misselt, K., Reach, W., Shiao, B., Tielens, X., Carlson, L., Churchwell, E., Clayton, G. C., Chen, C.-H. R., Cohen, M., Fukui, Y., Gorjian, V., Hony, S., Israel, F. P., Kawamura, A., Kemper, F., Leroy, A., Li, A., Madden, S., Marble, A. R., McDonald, I., Mizuno, A., Mizuno, N., Muller, E., Oliveira, J. M., Olsen, K., Onishi, T., Paladini, R., Paradis, D., Points, S., Robitaille, T., Rubin, D., Sandstrom, K., Sato, S., Shibai, H., Simon, J. D., Smith, L. J., Srinivasan, S., Vijh, U., Van Dyk, S., van Loon, J. T., and Zaritsky, 
D. 2011. Surveying the Agents of Galaxy Evolution in the Tidally Stripped, Low Metallicity Small Magellanic Cloud (SAGE-SMC). I. Overview. AJ, 142(Oct.), 102+ .

Gorlova, N., Van Winckel, H., Gielen, C., Raskin, G., Prins, S., Pessemier, W., Waelkens, C., Frémat, Y., Hensberge, H., Dumortier, L., Jorissen, A., and Van Eck, S. 2012. Time-resolved spectroscopy of BD+46 ${ }^{\mathrm{deg}} 442$ : Gas streams and jet creation in a newly discovered evolved binary with a disk. $A \& A, \mathbf{5 4 2}$ (June), A27.

Gorlova, N., Van Winckel, H., Ikonnikova, N. P., Burlak, M. A., Komissarova, G. V., Jorissen, A., Gielen, C., Debosscher, J., and Degroote, P. 2015. IRAS 19135+3937: an SRd variable as interacting binary surrounded by a circumbinary disc. MNRAS, 451(Aug.), 2462-2478.

Han, Z., Podsiadlowski, P., Maxted, P. F. L., Marsh, T. R., and Ivanova, N. 2002. The origin of subdwarf B stars - I. The formation channels. MNRAS, 336(Oct.), 449-466.

Han, Z., Podsiadlowski, P., Maxted, P. F. L., and Marsh, T. R. 2003. The origin of subdwarf B stars - II. MNRAS, 341(May), 669-691.

Herwig, F. 2005. Evolution of Asymptotic Giant Branch Stars. ARA\&A, 43(Sept.), 435479.

Hillen, M., Verhoelst, T., Van Winckel, H., Chesneau, O., Hummel, C. A., Monnier, J. D., Farrington, C., Tycner, C., Mourard, D., ten Brummelaar, T., Banerjee, D. P. K., and Zavala, R. T. 2013. An interferometric study of the post-AGB binary 89 Herculis. I. Spatially resolving the continuum circumstellar environment at optical and nearIR wavelengths with the VLTI, NPOI, IOTA, PTI, and the CHARA Array. $A \& A$, 559(Nov.), A111.

Hillen, M., Menu, J., Van Winckel, H., Min, M., Gielen, C., Wevers, T., Mulders, G. D., Regibo, S., and Verhoelst, T. 2014. An interferometric study of the post-AGB binary 89 Herculis. II. Radiative transfer models of the circumbinary disk. $A \& A, \mathbf{5 6 8}$ (Aug.), A12.

Hillen, M., de Vries, B. L., Menu, J., Van Winckel, H., Min, M., and Mulders, G. D. 2015. The evolved circumbinary disk of AC Herculis: a radiative transfer, interferometric, and mineralogical study. A\&A, 578(June), A40.

Hillen, M., Kluska, J., Le Bouquin, J.-B., Van Winckel, H., Berger, J.-P., Kamath, D., and Bujarrabal, V. 2016. Imaging the dust sublimation front of a circumbinary disk. $A \& A$, 588(Apr.), L1.

Hillen, M., Van Winckel, H., Menu, J., Manick, R., Debosscher, J., Min, M., de Wit, W.-J., Verhoelst, T., Kamath, D., and Waters, L. B. F. M. 2017. A mid-IR interferometric survey with MIDI/VLTI: resolving the second-generation protoplanetary disks around post-AGB binaries. A\&A, 599(Mar.), A41.

Hrivnak, B. J., Lu, W., Bohlender, D., Morris, S. C., Woodsworth, A. W., and Scarfe, C. D. 2011. Are Proto-planetary Nebulae Shaped by a Binary? Results of a Long-term Radial Velocity Study. ApJ, 734(June), 25.

Hrivnak, B. J., Van de Steene, G., Van Winckel, H., Sperauskas, J., Bohlender, D., and Lu, W. 2017. Where are the Binaries? Results of a Long-term Search for Radial Velocity Binaries in Proto-planetary Nebulae. ApJ, 846(Sept.), 96.

Ivanova, N., Justham, S., Chen, X., De Marco, O., Fryer, C. L., Gaburov, E., Ge, H., Glebbeek, E., Han, Z., Li, X.-D., Lu, G., Marsh, T., Podsiadlowski, P., Potter, A., Soker, N., Taam, R., Tauris, T. M., van den Heuvel, E. P. J., and Webbink, R. F. 2013. Common envelope evolution: where we stand and how we can move forward. $A \& A R v$, 21(Feb.), 59. 
Izzard, R. G., Hall, P. D., Tauris, T. M., and Tout, C. A. 2012 (Aug.). Common envelope evolution. Pages 95-102 of: IAU Symposium. IAU Symposium, vol. 283.

Jones, D., and Boffin, H. M. J. 2017. Binary stars as the key to understanding planetary nebulae. Nature Astronomy, 1(May), 0117.

Jura, M., Turner, J., and Balm, S. P. 1997. Big Grains in the Red Rectangle? ApJ, 474(Jan.), 741-+.

Kamath, D., Wood, P. R., and Van Winckel, H. 2014. Optically visible post-AGB/RGB stars and young stellar objects in the Small Magellanic Cloud: candidate selection, spectral energy distributions and spectroscopic examination. MNRAS, 439(Apr.), 2211-2270.

Kamath, D., Wood, P. R., and Van Winckel, H. 2015. Optically visible post-AGB stars, post-RGB stars and young stellar objects in the Large Magellanic Cloud. MNRAS, 454(Dec.), 1468-1502.

Kamath, D., Wood, P. R., Van Winckel, H., and Nie, J. D. 2016. A newly discovered stellar type: dusty post-red giant branch stars in the Magellanic Clouds. A\&A, 586(Feb.), L5.

Kraus, S., Hofmann, K.-H., Menten, K. M., Schertl, D., Weigelt, G., Wyrowski, F., Meilland, A., Perraut, K., Petrov, R., Robbe-Dubois, S., Schilke, P., and Testi, L. 2010. A hot compact dust disk around a massive young stellar object. Nature, 466(July), 339-342.

Lodders, K. 2003. Solar System Abundances and Condensation Temperatures of the Elements. ApJ, 591(July), 1220-1247.

Lubow, S. H. 2010. Eccentricity growth rates of tidally distorted discs. MNRAS, 406(Aug.), 2777-2786.

Maas, T., Van Winckel, H., and Waelkens, C. 2002. RU Cen and SX Cen: Two strongly depleted RV Tauri stars in binary systems. The RV Tauri photometric b phenomenon and binarity. $A \& A, \mathbf{3 8 6}$ (May), 504-516.

Manick, R., Van Winckel, H., Kamath, D., Hillen, M., and Escorza, A. 2017. Establishing binarity amongst Galactic RV Tauri stars with a disc. $A \& A, \mathbf{5 9 7}(J a n)$, A129.

Meixner, M., Gordon, K. D., Indebetouw, R., Hora, J. L., Whitney, B., Blum, R., Reach, W., Bernard, J.-P., Meade, M., Babler, B., Engelbracht, C. W., For, B.-Q., Misselt, K., Vijh, U., Leitherer, C., Cohen, M., Churchwell, E. B., Boulanger, F., Frogel, J. A., Fukui, Y., Gallagher, J., Gorjian, V., Harris, J., Kelly, D., Kawamura, A., Kim, S., Latter, W. B., Madden, S., Markwick-Kemper, C., Mizuno, A., Mizuno, N., Mould, J., Nota, A., Oey, M. S., Olsen, K., Onishi, T., Paladini, R., Panagia, N., Perez-Gonzalez, P., Shibai, H., Sato, S., Smith, L., Staveley-Smith, L., Tielens, A. G. G. M., Ueta, T., Dyk, S. V., Volk, K., Werner, M., and Zaritsky, D. 2006. Spitzer Survey of the Large Magellanic Cloud: Surveying the Agents of a Galaxy's Evolution (SAGE). I. Overview and Initial Results. AJ, 132(Dec.), 2268-2288.

Menu, J., van Boekel, R., Henning, T., Leinert, C., Waelkens, C., and Waters, L. B. F. M. 2015. The structure of disks around intermediate-mass young stars from midinfrared interferometry. Evidence for a population of group II disks with gaps. $A \& A$, 581(Sept.), A107.

Min, M., Waters, L. B. F. M., de Koter, A., Hovenier, J. W., Keller, L. P., and MarkwickKemper, F. 2007. The shape and composition of interstellar silicate grains. $A \& A$, 462(Feb.), 667-676.

Molster, F. J., Waters, L. B. F. M., Tielens, A. G. G. M., Koike, C., and Chihara, H. 2002. Crystalline silicate dust around evolved stars. III. A correlations study of crystalline silicate features. $A \& A, \mathbf{3 8 2}$ (Jan.), 241-255. 
Mulders, G. D., and Dominik, C. 2012. Probing the turbulent mixing strength in protoplanetary disks across the stellar mass range: no significant variations. $A \& A, \mathbf{5 3 9}$ (Mar.), A9.

Nie, J. D., Wood, P. R., and Nicholls, C. P. 2012. Predicting the fate of binary red giants using the observed sequence E star population: binary planetary nebula nuclei and post-RGB stars. MNRAS, 423(July), 2764-2780.

Rao, S. S., and Giridhar, S. 2014. Chemical Compositions of RV Tauri Stars and Related Objects. Rev. Mex. Astron. Astrofis., 50(Apr.), 49-65.

Raskin, G., van Winckel, H., Hensberge, H., Jorissen, A., Lehmann, H., Waelkens, C., Avila, G., de Cuyper, J.-P., Degroote, P., Dubosson, R., Dumortier, L., Frémat, Y., Laux, U., Michaud, B., Morren, J., Perez Padilla, J., Pessemier, W., Prins, S., Smolders, K., van Eck, S., and Winkler, J. 2011. HERMES: a high-resolution fibre-fed spectrograph for the Mercator telescope. $A \& A, \mathbf{5 2 6}(\mathrm{Feb}$.), A69.

Reyniers, M., and Van Winckel, H. 2007. First detection of photospheric depletion in the Large Magellanic Cloud. A\&A, 463(Feb.), L1-L4.

Sahai, R., Morris, M., Sánchez Contreras, C., and Claussen, M. 2007. Preplanetary Nebulae: A Hubble Space Telescope Imaging Survey and a New Morphological Classification System. AJ, 134(Dec.), 2200-2225.

Shiber, S., Kashi, A., and Soker, N. 2017. Simulating the onset of grazing envelope evolution of binary stars. MNRAS, 465(Feb.), L54-L58.

Soker, N. 2017. Energizing the last phase of common-envelope removal. MNRAS, 471(Nov.), 4839-4843.

Thomas, J. D., Witt, A. N., Aufdenberg, J. P., Bjorkman, J. E., Dahlstrom, J. A., Hobbs, L. M., and York, D. G. 2013. Geometry and velocity structure of HD 44179's bipolar jet. MNRAS, 430(Apr.), 1230-1237.

Toonen, S., and Nelemans, G. 2013. The effect of common-envelope evolution on the visible population of post-common-envelope binaries. $A \& A, \mathbf{5 5 7}$ (Sept.), A87.

van Aarle, E., van Winckel, H., Lloyd Evans, T., Ueta, T., Wood, P. R., and Ginsburg, A. G. 2011. The optically bright post-AGB population of the LMC. A\&A, 530(June), A90.

Van Winckel, H. 2003. Post-Agb Stars. ARA\&A, 41, 391-427.

Van Winckel, H., Waelkens, C., and Waters, L. B. F. M. 1995. The extremely iron-deficient "Post-AGB" stars and binaries. A\&A, 293(Jan.), L25-L28.

Van Winckel, H., Lloyd Evans, T., Briquet, M., De Cat, P., Degroote, P., De Meester, W., De Ridder, J., Deroo, P., Desmet, M., Drummond, R., Eyer, L., Groenewegen, M. A. T., Kolenberg, K., Kilkenny, D., Ladjal, D., Lefever, K., Maas, T., Marang, F., Martinez, P., Østensen, R. H., Raskin, G., Reyniers, M., Royer, P., Saesen, S., Uytterhoeven, K., Vanautgaerden, J., Vandenbussche, B., van Wyk, F., Vučković, M., Waelkens, C., and Zima, W. 2009. Post-AGB stars with hot circumstellar dust: binarity of the lowamplitude pulsators. $A \& A, \mathbf{5 0 5}$ (Oct.), 1221-1232.

Venn, K. A., Puzia, T. H., Divell, M., Côté, S., Lambert, D. L., and Starkenburg, E. 2014. Searching for Dust around Hyper Metal Poor Stars. ApJ, 791(Aug.), 98.

Vos, J., Østensen, R. H., Marchant, P., and Van Winckel, H. 2015. Testing eccentricity pumping mechanisms to model eccentric long-period sdB binaries with MESA. A\&A, 579(July), A49.

Waelkens, C., Van Winckel, H., Waters, L. B. F. M., and Bakker, E. J. 1996. Variability and nature of the binary in the Red Rectangle nebula. $A \& A, 314$ (Oct.), L17-LL20.

Waters, L. B. F. M., Trams, N. R., and Waelkens, C. 1992. A scenario for the selective depletion of stellar atmospheres. A\&A, 262(Sept.), L37-L40. 